\title{
Catalog of 174 Binary Black Hole Simulations for Gravitational Wave Astronomy
}

\author{
Abdul H. Mroué, ${ }^{1}$ Mark A. Scheel, ${ }^{2}$ Béla Szilágyi, ${ }^{2}$ Harald P. Pfeiffer, ${ }^{1,3}$ Michael Boyle, ${ }^{4}$ Daniel A. Hemberger, ${ }^{2,4}$ \\ Lawrence E. Kidder, ${ }^{4}$ Geoffrey Lovelace, ${ }^{5,2}$ Serguei Ossokine, ${ }^{1,6}$ Nicholas W. Taylor, ${ }^{2}$ Anıl Zenginoğlu, ${ }^{2}$ \\ Luisa T. Buchman, ${ }^{2}$ Tony Chu, ${ }^{1}$ Evan Foley, ${ }^{5}$ Matthew Giesler, ${ }^{5}$ Robert Owen, ${ }^{7}$ and Saul A. Teukolsky ${ }^{4}$ \\ ${ }^{1}$ Canadian Institute for Theoretical Astrophysics, 60 St. George Street, University of Toronto, Toronto, Ontario M5S 3H8, Canada \\ ${ }^{2}$ Theoretical Astrophysics 350-17, California Institute of Technology, Pasadena, California 91125, USA \\ ${ }^{3}$ Canadian Institute for Advanced Research, 180 Dundas Street West, Toronto, Ontario M5G 1Z8, Canada \\ ${ }^{4}$ Center for Radiophysics and Space Research, Cornell University, Ithaca, New York 14853, USA \\ ${ }^{5}$ Gravitational Wave Physics and Astronomy Center, California State University Fullerton, Fullerton, California 92834, USA \\ ${ }^{6}$ Department of Astronomy and Astrophysics, 50 St. George Street, University of Toronto, Toronto, Ontario M5S 3H4, Canada \\ ${ }^{7}$ Department of Physics and Astronomy, Oberlin College, Oberlin, Ohio 44074, USA
}

(Received 27 May 2013; revised manuscript received 5 September 2013; published 11 December 2013)

This Letter presents a publicly available catalog of 174 numerical binary black hole simulations following up to 35 orbits. The catalog includes 91 precessing binaries, mass ratios up to 8:1, orbital eccentricities from a few percent to $10^{-5}$, black hole spins up to $98 \%$ of the theoretical maximum, and radiated energies up to $11.1 \%$ of the initial mass. We establish remarkably good agreement with postNewtonian precession of orbital and spin directions for two new precessing simulations, and we discuss other applications of this catalog. Formidable challenges remain: e.g., precession complicates the connection of numerical and approximate analytical waveforms, and vast regions of the parameter space remain unexplored.

DOI: 10.1103/PhysRevLett.111.241104

PACS numbers: 04.25.dg, 04.25.Nx, 04.30.-w

Introduction.-Gravitational waves (GWs) from coalescing compact-object binaries-neutron stars (NS) and stellar-mass black holes $(\mathrm{BH})$ - are primary targets for next-generation GW detectors, such as Advanced LIGO, Virgo, and KAGRA [1-4]. Detecting GWs from compactobject binaries requires high-quality, accurate theoretical waveform models for GW template banks. Similarly, measuring source properties of detected signals ("parameter estimation") relies on theoretical waveform models used in Markov chain Monte Carlo algorithms [5].

For widely separated binaries, post-Newtonian (PN) calculations [6] provide accurate gravitational waveforms. However, numerical simulations of the full Einstein equations are needed during the late inspiral, merger, and ringdown. Such simulations are particularly important for stellar-mass BH-BH and BH-NS systems: Late inspirals and mergers occur near LIGO's most sensitive frequency range, and although $\mathrm{BHs}$ might have high spins $[7,8]$, some of the spin contributions to the PN waveforms are known only to lower expansion order than the nonspinning terms (Ref. [9] and references therein).

This Letter focuses on binary black holes (BBH). BBH simulations became possible eight years ago [10], with tremendous progress since (e.g., Refs. [11,12]). For best utility to GW astronomy, such simulations must achieve (i) sufficient accuracy, (ii) a desired orbital eccentricity, for instance, the very small eccentricity expected for field binaries [13,14], (iii) sufficient length (i.e., number of orbits) to connect reliably to PN waveforms, and (iv) sufficiently dense coverage of relevant regions of parameter space.
Satisfying all conditions (i) to (iv) is so difficult that simulations have barely reached the minimal desired quality. GW detection [condition (iii)] requires ten orbits for straightforward simulations (mass ratio $q \leqq 4$, dimensionless spins $\chi \equiv S / M^{2} \lesssim 0.7$ aligned with the orbital angular momentum) [15]; parameter estimation can benefit from well over 100 orbits [15-18]. These numbers increase with more extreme mass ratio and $\mathrm{BH}$ spins [17], and analogous estimates have not even been performed for precessing binaries. Yet, few published simulations follow even ten orbits.

Additionally, the seven-dimensional parameter space has barely been explored. The worldwide NINJA-2 Collaboration [19] yielded 40 waveforms with an average length of $\sim 9$ orbits, covering two one-dimensional subspaces of the aligned-spin region of the parameter space. The NRAR Collaboration [20] presented 25 waveforms of average length $\sim 13$ orbits and better accuracy than NINJA-2. Pekowsky et al. [21] report about 80 simulations covering approximately $7-12$ orbits $(\sim 25$ of them representing precessing binaries) and $\sim 170$ simulations lasting a few orbits each.

This Letter represents a major advance in waveform length and parameter-space coverage. We follow our earlier approach [22-25] of computing BBH simulations that are longer than previous simulations, with higher accuracy and very low eccentricity. Our catalog contains 174 simulations (155 are new and previously unpublished). 167 simulations cover more than 12 orbits (up to 35.5 orbits; see Fig. 2), and 91 represent precessing binaries. 


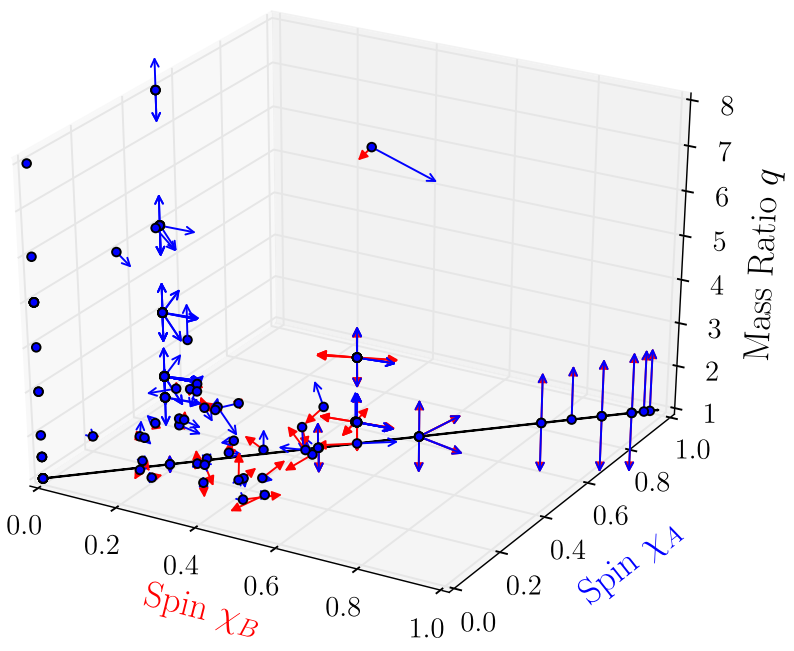

FIG. 1 (color online). The parameter space covered by our catalog. Each point represents a simulation. The axes show the mass ratio and the spin magnitudes of the larger (blue arrows) and smaller (red arrows) BHs. Arrows indicate the magnitude and direction of the spins.

This simulation catalog is publicly available [26] and will have other applications besides its primary goal of facilitating $\mathrm{GW}$ astronomy.

Techniques.-The simulations are computed using the Spectral Einstein Code (SpEC) [27]. Quasiequilibrium [28,29] initial data are constructed [30] to solve the Einstein constraint equations [31] for binaries with low $\left(\sim 10^{-4}\right)$ eccentricity [32-34] and are evolved using a generalized harmonic formulation [35-38] of Einstein's equations and damped harmonic gauge [39-41]. The adaptively refined [42] grid extends from pure-outflow excision boundaries conforming to the shapes of the apparent horizons $[24,41,43,44]$ to an artificial outer boundary where constraint-preserving boundary conditions $[38,45,46]$ are imposed. After the merger, the grid has only a single excision boundary $[24,43]$. Gravitational wave extraction and extrapolation of waveforms to infinity are described in Refs. [25,32,47-51].

No fine-tuning or trial and error is required for our current evolution method, for regions of parameter space covered by this catalog. Mesh refinement and grid transitions (such as regridding to a single excision boundary at merger) are automatic. This enables the completion of many simulations with little cost in human time.

Catalog.-Figure 1 represents the parameter-space coverage. The catalog includes (i) 62 new single-spin simulations with $\chi_{A}=0.5$ and $\chi_{B}=0$ ranging from $q=1$ to $q=8$, which extend efforts by the NINJA-2 Collaboration [19] (which contains only three unequal mass, aligned-spin simulations, and no precessing simulations) and the NRAR Collaboration [20] (which contains spinning simulations only for $q \leq 3$ ), (ii) 32 new simulations with random mass ratios $q \in[1,2]$ and random spins $\left(\chi_{A}, \chi_{B} \leq 0.5\right)$, (iii) 16 $q=1$ simulations with equal, aligned spins [23,52,53], including one new simulation with the highest $\mathrm{BH}$ spin to date $\left(\chi_{A}=\chi_{B}=0.98\right)$, (iv) a high mass ratio, high-spin $\left(q=6, \chi_{A}=0.9, \chi_{B}=0.3\right)$, precessing simulation intended to test numerical capabilities, and (v) 32 nonspinning simulations, including mass ratios $q=1.5,5,8$, not considered in Ref. [22]. Also included are new simulations of different orbital eccentricities and durations, which facilitate calculation of periastron advance [54] and efficient initial data generation [34].

While we generally reduce orbital eccentricity [33] to $e \leqq 10^{-4}$, the catalog contains 43 new simulations with eccentricities from $e<10^{-4}$ up to $e=0.06$, representing

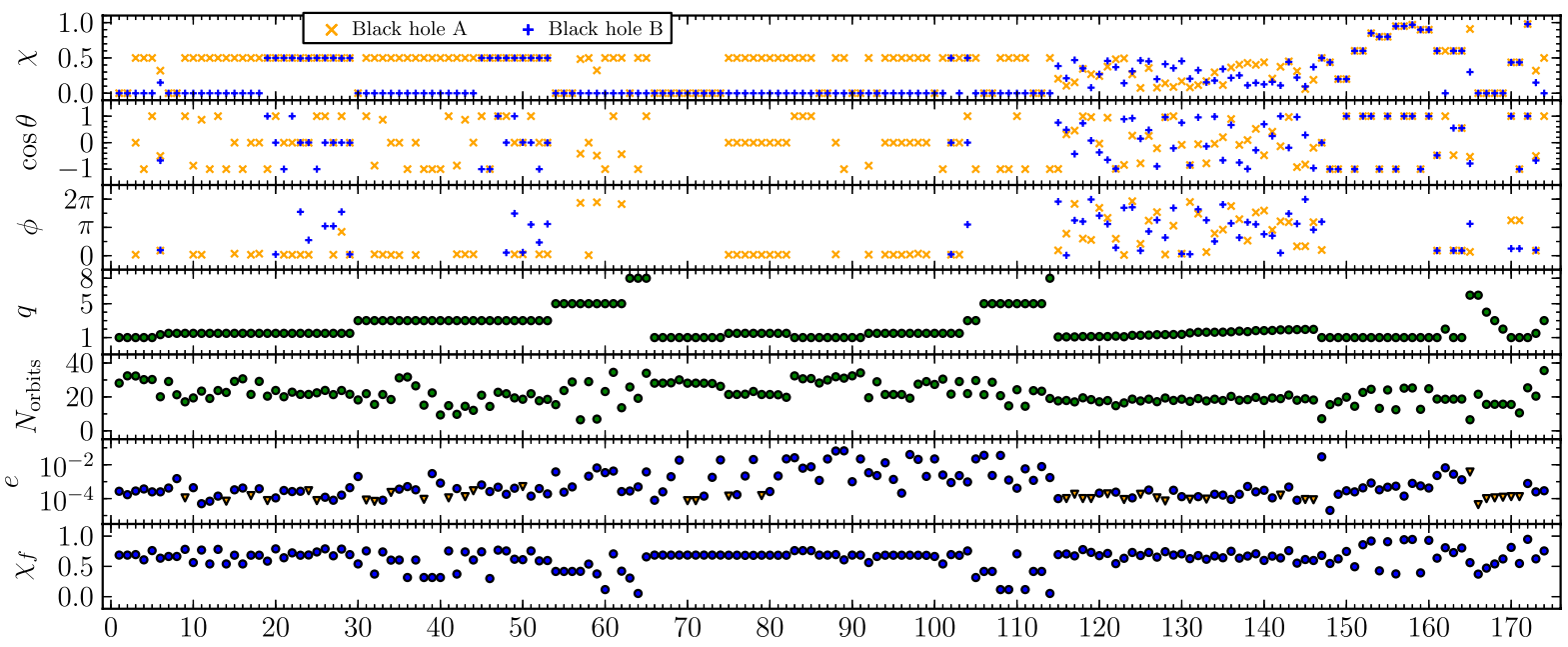

FIG. 2 (color online). Properties of all simulations in the catalog. From top to bottom: Dimensionless initial spin magnitudes $\chi_{A, B}$, angles $\theta_{A, B}$ between the initial spin vectors and the initial orbital angular momentum, angles $\phi_{A, B}$ between the line segment connecting the centers of the black holes and the initial spin vectors projected into the initial orbital plane, mass ratio $q=M_{A} / M_{B}$, number of orbits before merger, initial eccentricity $e$ (triangles indicate an upper bound on $e$ ), and final remnant spin $\chi_{f}$. 


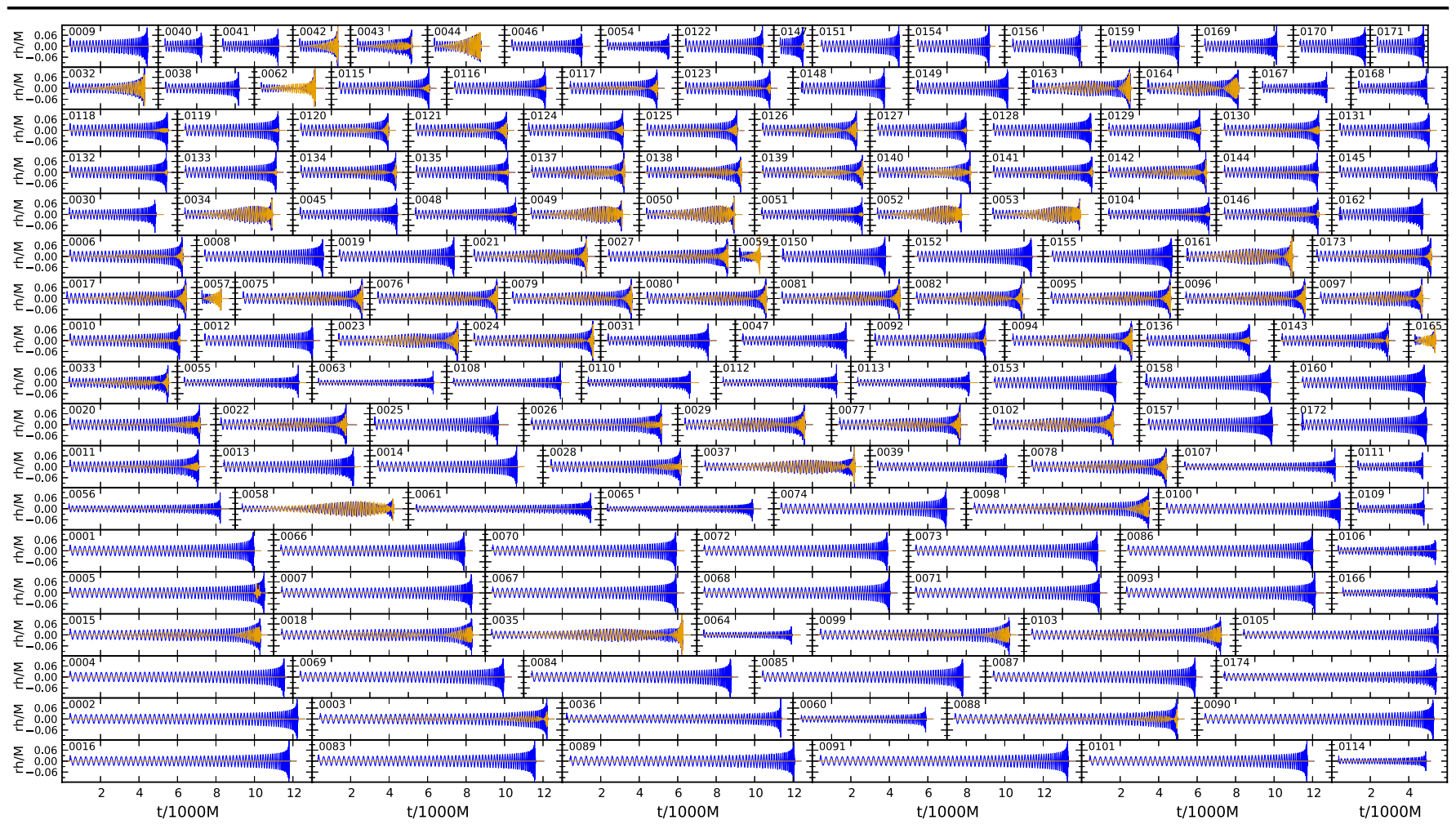

FIG. 3 (color online). Waveform polarizations $(r / M) h_{+}$(blue curves) and $(r / M) h_{\times}$(orange curves) in a sky direction parallel to the initial orbital plane of each simulation. The unit of the time axis corresponds to $1000 M=0.1 \mathrm{~s}$ for binaries with total mass $M=20 M_{\odot}$.

the first systematic sampling of slightly eccentric BBH waveforms.

Figure 3 plots the gravitational wave polarizations $h_{+}$ and $h_{\times}$emitted into a certain sky direction, chosen so that $h_{\times}$vanishes for nonprecessing systems.

Figure 4 shows the waveforms for simulation 0035 ( $q=3$, precessing, 31 orbits) in two sky directions, highlighting the waveforms' strong dependence on the $\mathrm{BBH}$ orientation relative to the line of sight to Earth. Figure 4 also presents a convergence test, showing differences in the waveforms computed using different numerical resolutions. We reach cumulative phase errors similar to shorter SpEC simulations [22,24,25], which were instrumental for various GW data-analysis applications $[19,48,55,56]$. The achieved error is about an order of magnitude smaller than the NRAR project's target [20] and is suitable for detection and parameter estimation. This accuracy is representative for all simulations; a more detailed discussion will be presented in Ref. [57].

Discussion.-This Letter presents the most comprehensive catalog of high-quality BBH simulations to date, enabling studies to help maximize the impact of $\mathrm{GW}$ detectors and to increase our understanding of GW sources and dynamical, strongly curved spacetime.

Accuracy of PN precession equations: PN theory predicts how spin and orbital angular momenta precess in generic binaries (e.g., Ref. [58]). The simulations here are long enough for detailed comparisons at different points in parameter space. As an example, Fig. 5 highlights two precessing systems. (i) Simulation 0035 (cf. Fig. 4) proceeds through about 1.5 precession cycles: the normal to the instantaneous orbital plane traces out the red precession cone with opening angle $35^{\circ}$, whereas the spin of

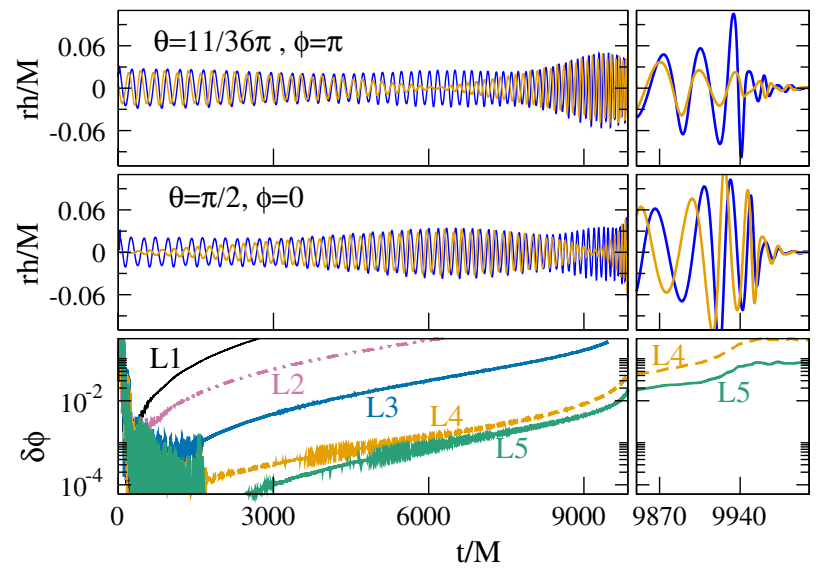

FIG. 4 (color online). Top: Waveforms $r h_{+} / M$ (blue curves) and $r h_{\times} / M$ (orange curves) for two detector directions $(\theta, \phi)$ relative to the initial orbital plane. Bottom: Cumulative phase differences between the $\ell=2, m=2$ spherical-harmonic mode of the waveform of numerical resolutions $L 1$ through $L 5$ compared to that of the highest resolution $L 6$. 

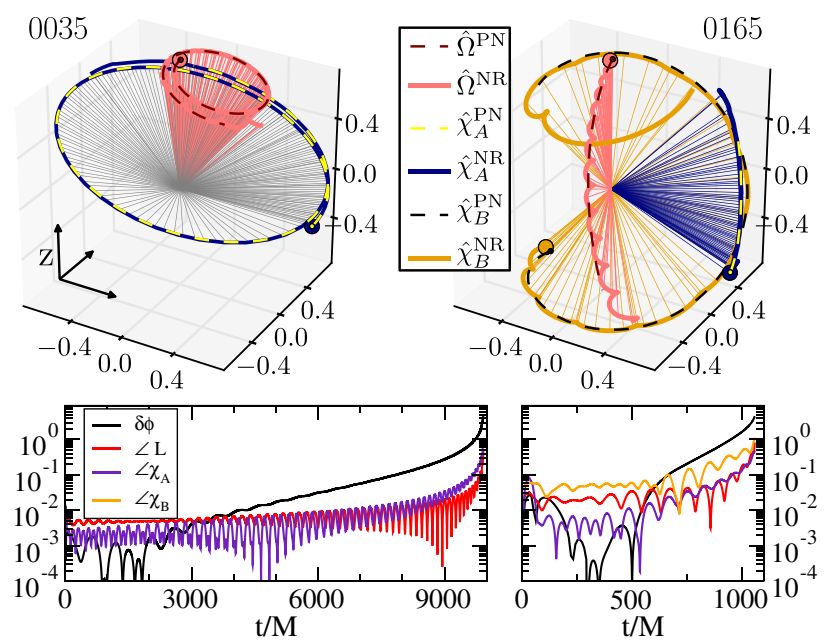

FIG. 5 (color online). Precessing simulations 0035 and 0165. Top: Precession and nutation of the orbital angular momentum and the spin vectors of the BHs. The circles denote the initial location of the vectors. Bottom: Comparison of dynamical quantities with post-Newtonian theory: angle $\angle L$ between PN and NR Newtonian orbital angular momentum, angle $\angle \chi_{A, B}$ between PN and NR spins $\left(\chi_{B}=0\right.$ for 0035$)$, and difference $\delta \phi_{\text {orb }}$ in orbital phase. All differences are in radians.

the more massive $\mathrm{BH}$ traces out a precession cone with an opening angle of $144^{\circ}$. (ii) Simulation 0165 has $q=6$ and spin magnitudes $\chi_{A}=0.9$ and $\chi_{B}=0.3$ in generic directions. The orbital plane changes by almost $90^{\circ}$, and the spin direction of the smaller BH traces out a spiral motion. The orbital angular momentum and spin vectors computed from the most recent available PN approximant [9] (dashed lines) agree well with numerical relativity (NR) until the end of the inspiral. The lower panel of Fig. 5 quantifies this agreement. Details of this study will be discussed in Ref. [59].

Precessing inspiral-merger-ringdown waveform models [60-62] can be tested and improved with the catalog, and new models can be developed. Some of these models require knowledge of radiated energy; we investigate this in Fig. 6. More energy is radiated at lower mass ratios, and the simulation with the most radiated energy $(11.1 \%$, the highest to date for BBH inspirals) is new, having the highest black hole spins $(\chi=0.98)$ to date. This radiated energy

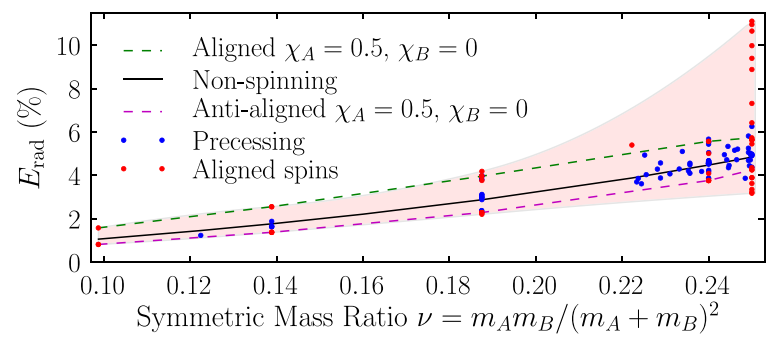

FIG. 6 (color online). The radiated energy (as measured by the fractional difference between the initial and final Christodoulou masses, where the initial mass is the sum of the holes' masses after the initial relaxation) for each simulation in the catalog. is larger than the maximum predicted by previous phenomenological fits (compare with Refs. [63,64]) but agrees well with the recent fitting formula of Ref. [53], which includes simulations with spins up to $\chi=0.97$. To our knowledge, no previous remnant mass calculations have been performed for spinning BBHs at mass ratios $q>4$.

Periastron advance can be studied in aligned-spin binaries and generic binaries [54,65,66], using simulations with different eccentricities. This allows the first calculation of the gravitational self-force contribution to the periastron advance for spinning binaries [67].

PN accuracy studies, extended to a larger region of parameter space and to an earlier stage of the inspiral: Previous studies consider only aligned spins $[23,68,69]$ and at best either $15-20$ orbits $[16,25]$ or a few orbits at large separation [70]. With the longer waveforms in this catalog, these studies can extend to the earlier part of the inspiral, where PN theory is expected to be more accurate and to include a larger region of parameter space.

Independent validation of existing analytical waveform models: Many waveform models [49,71-75] are calibrated against numerical relativity simulations-but usually only with a small number of short (typically $<10$ orbits) simulations. The new simulations here enable tests of these models at many different points in parameter space and covering more cycles.

Detection sensitivity: Following the approach taken in the NINJA projects $[19,56,76]$, our waveforms can be injected into GW detector noise to study the efficiency of GW dataanalysis pipelines. Injections of precessing and/or eccentric waveforms from this catalog can quantify the impact of precession and eccentricity on the detection sensitivity of current searches using circular, aligned-spin templates. The new waveforms will also help assess the performance of searches with precessing waveform templates.

Systematic errors in parameter estimation: Parameter estimation methods [5] currently use inspiral-only PN waveforms. Applying parameter estimation methods to the waveforms in this catalog will enable the systematic errors of this approach to be quantified.

While this catalog will enable pioneering studies, major challenges remain for future work. First, for a waveform to be most useful for data analysis, it must be connected to a PN waveform from the early inspiral, forming a hybrid waveform [19] that spans the entire frequency range of a $\mathrm{GW}$ detector. This is difficult for precessing configurations because of both the complexity of precessing PN waveforms and ambiguities in connecting PN binary parameters with the numerical binary parameters [51].

Second, most of the parameter space remains unexplored. Only 24 configurations have mass ratio $q>3$ (cf. Fig. 2); of these, only five are precessing and almost none have a spinning smaller black hole. Spinning BBH systems for $5 \lessgtr q \lesssim 10$ are particularly interesting because they may serve as accurate proxies for BH-NS 
binaries [77]. Furthermore, the catalog contains only four simulations (the only four to date [23,53]) with spins $\chi \geq 0.93$.

Finally, for some simulations, the numerical resolution is determined by specifying the adaptive mesh refinement (AMR) tolerance. The times at which AMR makes adjustments depend on this tolerance, so comparing simulations with different AMR tolerances is not always as straightforward to interpret as Fig. 4. This will be discussed within a more comprehensive analysis of this catalog in Ref. [57]. The catalog is available for download [26].

We thank Christian Ott and Kip Thorne for helpful discussions. This work was supported in part by NSERC of Canada, the Canada Chairs Program, and the Canadian Institute for Advanced Research; the Sherman Fairchild Foundation; and NSF Grants No. PHY-0969111 and No. PHY-1005426 at Cornell, NSF Grants No. PHY1068881, No. PHY-1005655, and No. DMS-1065438 at Caltech, and NSF Grant No. PHY-1307489 at Cal State Fullerton. Simulations used in this work were computed with the SpEC code [27]. Computations were performed on the Zwicky cluster at Caltech, which is supported by the Sherman Fairchild Foundation and by NSF Grant No. PHY-0960291; on the NSF XSEDE network under Grant No. TG-PHY990007N; on the Orca Cluster supported by Cal State Fullerton; and on the GPC Supercomputer at the SciNet HPC Consortium [78]. SciNet is funded by the Canada Foundation for Innovation under the auspices of Compute Canada, the Government of Ontario, Ontario Research FundResearch Excellence, and the University of Toronto.

[1] G. M. Harry (LIGO Scientific Collaboration), Classical Quantum Gravity 27, 084006 (2010).

[2] F. Acernese et al. (Virgo Collaboration), Advanced Virgo Baseline Design, Report No. VIR-0027A-09, 2009, https://tds.ego-gw.it/q1/?c=6589.

[3] T. Accadia et al. (Virgo Collaboration), Advanced Virgo Technical Design, Report No. VIR-0128A-12, 2012, https://tds.ego-gw.it/ql/?c=8940.

[4] K. Somiya and (the KAGRA Collaboration), Classical Quantum Gravity 29, 124007 (2012).

[5] J. Aasi et al. (LIGO Scientific Collaboration and Virgo Collaboration), Phys. Rev. D 88, 062001 (2013).

[6] L. Blanchet, Living Rev. Relativity 9, 4 (2006).

[7] L. Gou, J. E. McClintock, M. J. Reid, J. A. Orosz, J. F. Steiner, R. Narayan, J. Xiang, R. A. Remillard, K. A. Arnaud, and S. W. Davis, Astrophys. J. 742, 85 (2011).

[8] J. E. McClintock, R. Shafee, R. Narayan, R. A. Remillard, S. W. Davis, and L.-X. Li, Astrophys. J. 652, 518 (2006).

[9] A. Bohe, S. Marsat, and L. Blanchet, Classical Quantum Gravity 30, 135009 (2013).

[10] F. Pretorius, Phys. Rev. Lett. 95, 121101 (2005).

[11] J. Centrella, J. G. Baker, B. J. Kelly, and J. R. van Meter, Rev. Mod. Phys. 82, 3069 (2010).
[12] H.P. Pfeiffer, Classical Quantum Gravity 29, 124004 (2012).

[13] P. C. Peters and J. Mathews, Phys. Rev. 131, 435 (1963).

[14] P. C. Peters, Phys. Rev. 136, B1224 (1964).

[15] F. Ohme, M. Hannam, and S. Husa, Phys. Rev. D 84, 064029 (2011).

[16] I. MacDonald, A. H. Mroué, H. P. Pfeiffer, M. Boyle, L. E. Kidder, M. A. Scheel, B. Szilágyi, and N. W. Taylor, Phys. Rev. D 87, 024009 (2013).

[17] M. Boyle, Phys. Rev. D 84, 064013 (2011).

[18] T. Damour, A. Nagar, and M. Trias, Phys. Rev. D 83, 024006 (2011).

[19] P. Ajith, M. Boyle, D. A. Brown, B. Brugmann, and L. T. Buchman et al., Classical Quantum Gravity 29, 124001 (2012).

[20] I. Hinder et al. (NRAR Collaboration), arXiv:1307.5307 [Classical Quantum Gravity (to be published)].

[21] L. Pekowsky, R. O'Shaughnessy, J. Healy, and D. Shoemaker, Phys. Rev. D 88, 024040 (2013).

[22] L. T. Buchman, H. P. Pfeiffer, M. A. Scheel, and B. Szilágyi, Phys. Rev. D 86, 084033 (2012).

[23] G. Lovelace, M. Boyle, M. A. Scheel, and B. Szilágyi, Classical Quantum Gravity 29, 045003 (2012).

[24] M. A. Scheel, M. Boyle, T. Chu, L. E. Kidder, K. D. Matthews, and H. P. Pfeiffer, Phys. Rev. D 79, 024003 (2009).

[25] M. Boyle, D. A. Brown, L. E. Kidder, A. H. Mroué, H.P. Pfeiffer, M. A. Scheel, G. B. Cook, and S. A. Teukolsky, Phys. Rev. D 76, 124038 (2007).

[26] http://www.black-holes.org/waveforms.

[27] http://www.black-holes.org/SpEC.html.

[28] M. Caudill, G. B. Cook, J. D. Grigsby, and H. P. Pfeiffer, Phys. Rev. D 74, 064011 (2006).

[29] G. Lovelace, R. Owen, H. P. Pfeiffer, and T. Chu, Phys. Rev. D 78, 084017 (2008).

[30] H. P. Pfeiffer, L. E. Kidder, M. A. Scheel, and S. A. Teukolsky, Comput. Phys. Commun. 152, 253 (2003).

[31] J. W. York, Phys. Rev. Lett. 82, 1350 (1999).

[32] H. P. Pfeiffer, D. A. Brown, L. E. Kidder, L. Lindblom, G. Lovelace, and M. A. Scheel, Classical Quantum Gravity 24, S59 (2007).

[33] A. Buonanno, L. E. Kidder, A. H. Mroué, H.P. Pfeiffer, and A. Taracchini, Phys. Rev. D 83, 104034 (2011).

[34] A. H. Mroué and H. P. Pfeiffer, arXiv:1210.2958.

[35] H. Friedrich, Commun. Math. Phys. 100, 525 (1985).

[36] D. Garfinkle, Phys. Rev. D 65, 044029 (2002).

[37] F. Pretorius, Classical Quantum Gravity 22, 425 (2005).

[38] L. Lindblom, M. A. Scheel, L. E. Kidder, R. Owen, and O. Rinne, Classical Quantum Gravity 23, S447 (2006).

[39] L. Lindblom and B. Szilágyi, Phys. Rev. D 80, 084019 (2009).

[40] M. W. Choptuik and F. Pretorius, Phys. Rev. Lett. 104, 111101 (2010).

[41] B. Szilágyi, L. Lindblom, and M. A. Scheel, Phys. Rev. D 80, 124010 (2009).

[42] G. Lovelace, M. A. Scheel, and B. Szilágyi, Phys. Rev. D 83, 024010 (2011).

[43] D. A. Hemberger, M. A. Scheel, L. E. Kidder, B. Szilágyi, G. Lovelace, N. W. Taylor, and S. A. Teukolsky, Classical Quantum Gravity 30, 115001 (2013). 
[44] S. Ossokine, L. E. Kidder, and H. P. Pfeiffer, Phys. Rev. D 88, 084031 (2013).

[45] O. Rinne, Classical Quantum Gravity 23, 6275 (2006).

[46] O. Rinne, L. Lindblom, and M.A. Scheel, Classical Quantum Gravity 24, 4053 (2007).

[47] O. Rinne, L. T. Buchman, M. A. Scheel, and H. P. Pfeiffer, Classical Quantum Gravity 26, 075009 (2009).

[48] Y. Pan, A. Buonanno, M. Boyle, L. T. Buchman, L.E. Kidder, H. P. Pfeiffer, and M. A. Scheel, Phys. Rev. D 84, 124052 (2011).

[49] A. Buonanno, Y. Pan, H. P. Pfeiffer, M. A. Scheel, L. T. Buchman, and L.E. Kidder, Phys. Rev. D 79, 124028 (2009).

[50] M. Boyle and A.H. Mroué, Phys. Rev. D 80, 124045 (2009).

[51] M. Boyle, Phys. Rev. D 87, 104006 (2013).

[52] T. Chu, H. P. Pfeiffer, and M. A. Scheel, Phys. Rev. D 80, 124051 (2009).

[53] D. A. Hemberger, G. Lovelace, T. J. Loredo, L. E. Kidder, M. A. Scheel, B. Szilágyi, N.W. Taylor, and S. A. Teukolsky, Phys. Rev. D 88, 064014 (2013).

[54] A.H. Mroué, H.P. Pfeiffer, L.E. Kidder, and S. A. Teukolsky, Phys. Rev. D 82, 124016 (2010).

[55] Y. Pan, A. Buonanno, M. Boyle, L. T. Buchman, L.E. Kidder, H. P. Pfeiffer, and M. A. Scheel, Phys. Rev. D 84, 124052 (2011).

[56] B. Aylott, J. G. Baker, W. D. Boggs, M. Boyle, and P. R. Brady et al., Classical Quantum Gravity 26, 165008 (2009).

[57] A. Mroué, M. Scheel, B. Szilagyi, H. Pfeiffer, M. Boyle, D. Hemberger, L. Kidder, G. Lovelace, S. Ossokine, and N. Taylor et al. (to be published).

[58] J. Hartung, J. Steinhoff, and G. Schäfer, Ann. Phys. (Berlin) 525, 359 (2013).

[59] S. Ossokine et al. (to be published).

[60] R. Sturani, S. Fischetti, L. Cadonati, G. M. Guidi, J. Healy, D. Shoemaker, and A. Viceré, J. Phys. Conf. Ser. 243, 012007 (2010).

[61] Y. Pan, A. Buonanno, A. Taracchini, L. E. Kidder, and A. H. Mroue et al., arXiv:1307.6232.

[62] M. Hannam, P. Schmidt, A. Boh, L. Haegel, and S. Husa et al., arXiv:1308.3271.
[63] C. Reisswig, S. Husa, L. Rezzolla, E. N. Dorband, D. Pollney, and J. Seiler, Phys. Rev. D 80, 124026 (2009).

[64] E. Barausse, V. Morozova, and L. Rezzolla, Astrophys. J. 758, 63 (2012).

[65] A. Le Tiec, A. H. Mroué, L. Barack, A. Buonanno, H. P. Pfeiffer, N. Sago, and A. Taracchini, Phys. Rev. Lett. 107, 141101 (2011).

[66] T. Hinderer, A. Buonanno, A. H. Mroué, D. A. Hemberger, G. Lovelace, H. P. Pfeiffer, L. E. Kidder, M. A. Scheel, B. Szilagy, and N. W. Taylor et al., Phys. Rev. D 88, 084005 (2013).

[67] A. Le Tiec, A. Buonanno, A. H. Mroué, D. A. Hemberger, G. Lovelace, H. P. Pfeiffer, L. E. Kidder, M. A. Scheel, B. Szilagy, and N.W. Taylor et al., arXiv:1309.0541 [Phys. Rev. D (to be published)].

[68] M. Hannam, S. Husa, F. Ohme, D. Muller, and B. Brugmann, Phys. Rev. D 82, 124008 (2010).

[69] M. Hannam, S. Husa, B. Brügmann, and A. Gopakumar, Phys. Rev. D 78, 104007 (2008).

[70] C. O. Lousto and Y. Zlochower, Phys. Rev. D 88, 024001 (2013).

[71] T. Damour, A. Nagar, M. Hannam, S. Husa, and B. Brugmann, Phys. Rev. D 78, 044039 (2008).

[72] P. Ajith, M. Hannam, S. Husa, Y. Chen, B. Bruegmann, N. Dorband, D. Mueller, F. Ohme, D. Pollney, and C. Reisswig et al., Phys. Rev. Lett. 106, 241101 (2011).

[73] Y. Pan, A. Buonanno, L. T. Buchman, T. Chu, L.E. Kidder, H. P. Pfeiffer, and M. A. Scheel, Phys. Rev. D 81, 084041 (2010).

[74] L. Santamaría, F. Ohme, P. Ajith, B. Brügmann, N. Dorband, M. Hannam, S. Husa, P. Mösta, D. Pollney, and C. Reisswig et al., Phys. Rev. D 82, 064016 (2010).

[75] A. Taracchini, Y. Pan, A. Buonanno, E. Barausse, M. Boyle, T. Chu, G. Lovelace, H. P. Pfeiffer, and M. A. Scheel, Phys. Rev. D 86, 024011 (2012).

[76] L. Cadonati et al., Classical Quantum Gravity 26, 114008 (2009).

[77] F. Foucart, L. Buchman, M. D. Duez, M. Grudich, L. E. Kidder, I. MacDonald, A. Mroue, H. P. Pfeiffer, M. A. Scheel, and B. Szilagyi, Phys. Rev. D 88, 064017 (2013).

[78] C. Loken, D. Gruner, L. Groer, R. Peltier, N. Bunn, M. Craig, T. Henriques, J. Dempsey, C.-H. Yu, and J. Chen et al., J. Phys. Conf. Ser. 256, 012026 (2010). 